\title{
Antihyperon Polarization in Inclusive Processes at High Energies
}

\author{
C. C. Barros, Jr. and Y. Hama \\ Instituto de Física, Universidade de São Paulo, C.P. 66318, 05315-970, São Paulo, SP, Brazil
}

Received on 15 August, 2003.

\begin{abstract}
We propose a model that we believe is the main source of the antihyperon polarization in high-energy protonnucleus inclusive reactions. The polarization is originated by the final-state interactions between the antihyperons and other produced particles in these collisions (predominantly pions). The model is based on two elements: the low-energy pion-hyperon interaction (described by chiral effective Lagrangians) and the statistical fluctuations plus expansion of the background matter.
\end{abstract}

In 1976, Bunce and collaborators [1], studying the $\Lambda$ inclusive production

$$
p+B e \rightarrow \Lambda+X
$$

with $200 \mathrm{GeV}$ protons, where $X$ represents the not observed particles, verified that the $\Lambda$ polarization was significantly different from zero (and negative, reaching values close to $20 \%$ ). These results were totally unexpected [2], because there were indications that polarization effects should vanish at high energies and hadron polarization was believed to be a low energy phenomenon.

Since then, many similar experiments have been performed [3]-[6], that confirmed these results and have shown also that both hyperons and antihyperons produced are polarized in this kind of reaction. However, the variety of behavior of the polarizations has caused many problems to the theoretical understanding of the experimental data ([7]-[9] for example), specially in the antihyperon case. Since these models are based in direct mechanisms for hyperon production (the initial proton becomes a hyperon, by some mechanism, such as quark recombination [7], for example) the final antihyperon polarization practically vanishes.

The first model that was able to produce polarized antihyperons, was the one proposed by Y. Hama and T. Kodama [10] and it was based in the hydrodynamical model. The hyperon is produced inside a medium composed of hot hadronic matter (produced in the collision process) and interacts with it. This interaction was represented by an optic potential. With this model, the antihyperon data were quite well reproduced. Nevertheless, the model left some questions in open, such as the need of different potentials for different hyperons and their interpretations.

Aiming to study these questions, a new model was developed, [11], based in [10]. The hyperon was considered to be produced unpolarized (as an average effect) in the interior of a hot hadronic matter, now considered as a fluid composed of particles (predominantly pions). The hyperon (or antihyperon) interacts with the other hadrons (instead of considering a potential as it was done in [10]), and becomes polarized due to these final state interactions. The remainder of this work will be devoted to the presentation of the main features of this model, and the results of the $\bar{\Lambda}$ and $\bar{\Xi}^{+}$ polarizations (the calculations to the $\bar{\Sigma}^{-}$polarization are in progress).

To build a model with the cited characteristics, two elements must be considered: the microscopic interactions, that happen inside the hadronic matter, and the calculation of averages, considering the fluid in expansion. A brief description of these elements will be made below.

Currently, it is well known that models based on chiral Lagrangians describe very accurately many low energy hadronic processes, as for example the $\pi N$ interactions [12]. Thus, this seems to be the most reliable treatment to be followed in studying the low energy $\pi Y$ interactions [13].

The most important processes in the $\pi \Lambda, \pi \Sigma$ and $\pi \Xi$ interactions (at low energies) are shown in the Figs. 1, 2 and 3. The expressions resulting from the calculations of the diagrams (and a review of the formalism) may be found in [13], where the sigma term was introduced simply as a parametrization. In recent studies [12], [14], it was shown that the $\sigma$ term may be understood in terms of loops of pions, and then, in the calculation of the diagrams $1 \mathrm{c}, 2 \mathrm{c}$ and $3 c$, the expressions from [15], [16] have been used.

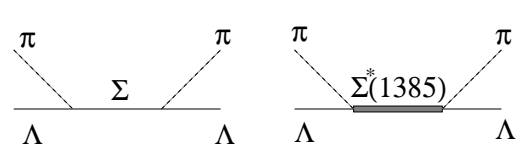

Figure 1. $\pi \Lambda$ interaction.
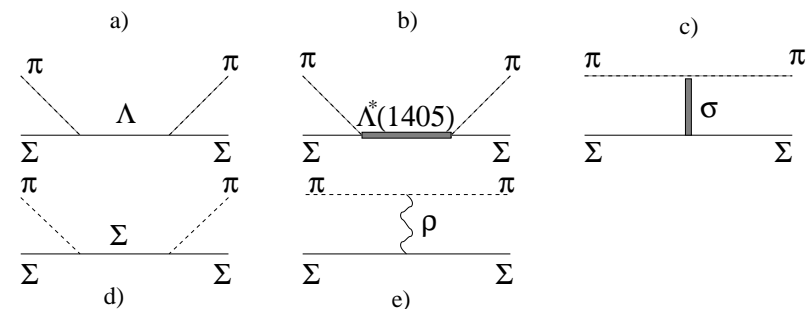

Figure 2. $\pi \Sigma$ interaction. 


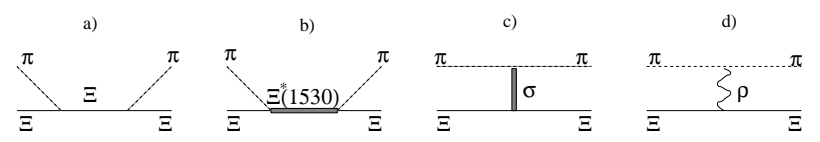

Figure 3. $\pi \Xi$ interaction.

Calculating the diagrams of the Figs. 1-3, we obtain a scattering matrix of the form [13]

$$
M_{I}^{b a}=\frac{T_{I}^{b a}}{8 \pi \sqrt{s}}=f_{I}(\theta)+\vec{\sigma} \cdot \hat{n} g_{I}(\theta)
$$

from which we can calculate the polarizations and the angular distributions with

$$
\begin{aligned}
& \frac{d \sigma}{d \Omega}=\frac{1}{k^{2}}\left\{|f|^{2}+|g|^{2}\right\}, \\
& \vec{P}=-2 \frac{\operatorname{Im}\left(f^{*} g\right)}{|f|^{2}+|g|^{2}} \hat{n} .
\end{aligned}
$$

Now, let us turn our attention to the second element of the model, that is to determine the average effect of the elementary interactions in high energy processes, using a hydrodynamical treatment. In fact, there are two averages to be made. The first one is in relation to a fluid element. The particles, produced thermally inside a fluid element interact, then become polarized. The second average is necessary due to the fact that the fluid is in expansion, so, their elements have different velocities. The transverse expansion effect [17], [18] will also be considered, because, as it is well known, it is an important characteristic of a hydrodynamical description of high energy hadronic collisions.

For this purpose, the idea is to suppose that in addition to the longitudinal expansion, that will obey a rapidity distribution $d N / d \alpha$ (where $\alpha$ is the longitudinal rapidity of the fluid element), the fluid has also a transverse expansion with a rapidity $\alpha_{t}$ of this element, that obeys another distribution, $d N / d \alpha_{t}$

Consequently, the average polarization may be calculated by the expression

$$
\langle\vec{P}\rangle=\frac{\int\left\{\left(\vec{P} \frac{d \sigma}{d t}\right)_{R_{1}}+\ldots+\left(\vec{P} \frac{d \sigma}{d t}\right)_{R_{N}}\right\} \mathcal{G} d \tau}{\int\left\{\left(\frac{d \sigma}{d t}\right)_{R_{1}}+\ldots+\left(\frac{d \sigma}{d t}\right)_{R_{N}}\right\} \mathcal{G} d \tau},
$$

where the $R_{i}$ reactions, elastic and with charge exchange, are considered, in the interactions of the hyperons with $\pi^{+}, \pi^{-}$and $\pi^{0}$ (the considered reactions are listed in [13]). These particles are produced with initial momenta $\vec{\pi}_{0}^{\prime}$ (pions) and $\vec{\Lambda}_{0}^{\prime}$ (hyperons) obeying statistical distributions (Fermi-Dirac and Bose-Einstein). The hyperon emerges with final momentum $\vec{\Lambda}^{\prime}$ and the energies are respectively $E_{\pi_{0}}^{\prime}, E_{0}^{\prime}$ e $E^{\prime}$. Then, the factor $\mathcal{G}$ can be written as

$$
\begin{aligned}
\mathcal{G}=\quad & \frac{\left(d^{2} N / d \alpha d \alpha_{t}\right)}{\left(\exp \left(\frac{E_{\pi_{0}}^{\prime}}{T}\right)-1\right)\left(\exp \left(\frac{E_{0}^{\prime}}{T}\right)+1\right)} \Lambda_{0}^{\prime 2} \pi_{0}^{\prime 2} \\
& \times \delta\left(E_{0}^{\prime}+E_{\pi_{0}}^{\prime}-E^{\prime}-\sqrt{m_{\pi}^{2}+\left(\vec{\pi}_{0}^{\prime}+\vec{\Lambda}_{0}^{\prime}-\vec{\Lambda}^{\prime}\right)^{2}}\right),
\end{aligned}
$$
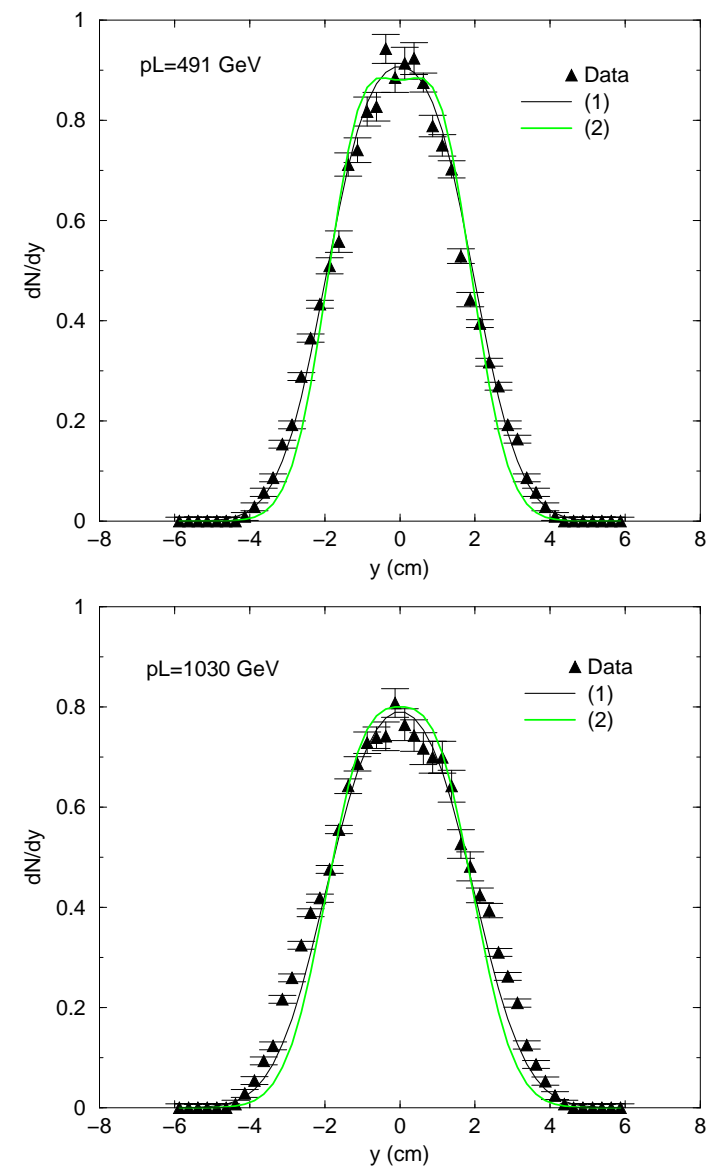

Figure 4. Parametrization of $d \sigma / d \alpha$ compared with the data from [19]. (1) is calculated with the text parameters, and (2), with $\beta=5$.

and the integration element is

$$
d \tau=d \alpha d \alpha_{t} d \vec{\Lambda}_{0}^{\prime} d \vec{\pi}_{0}^{\prime}
$$

In order to calculate the average polarization (5), we must know the forms of the rapidity distributions of the fluid elements. It is possible to obtain these distributions, by considering the pion rapidity distribution

$$
\frac{d \sigma}{d y}=\int \frac{d \sigma}{d \alpha}(\alpha) \frac{d \sigma}{d y^{\prime}}(y-\alpha) d \alpha,
$$

as a convolution of the rest-frame rapidity distribution $d \sigma / d y^{\prime}$, and the fluid rapidity distribution, $d \sigma / d \alpha$ (that we want to know).

The momentum distribution with respect to the fluid element (considering the thermal production of pions) is

$$
\frac{d \sigma}{d \vec{p}_{\pi}}=\frac{1}{E_{\pi}} \frac{d \sigma}{d y^{\prime} d \vec{p}_{t}}=\frac{1}{e^{\frac{E_{\pi}}{T}}-1}
$$

that after some manipulations [11] gives

$$
\frac{d \sigma}{d y^{\prime}} \sim C e^{-\beta^{\prime} y^{\prime}}
$$



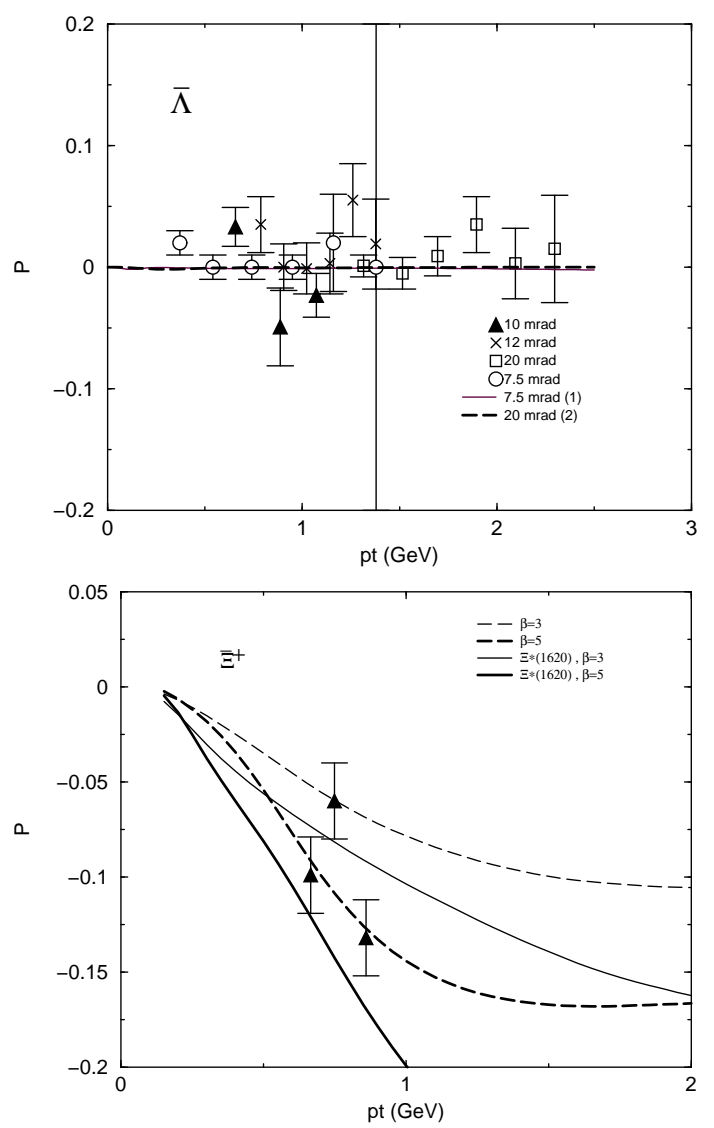

Figure 5. $\bar{\Lambda}$ and $\bar{\Xi}$ polarizations, with variations of the $\beta$ parameter (fluid rapidity distribution) and verifying the effect of the resonance $\Xi^{*}(1620)$.

with $\beta^{\prime} \sim 0.62$.

Supposing that $d \sigma / d \alpha$ may be described by a sum of gaussian functions

$$
\frac{d \sigma}{d \alpha}=A\left[e^{-\beta\left(\alpha-\alpha_{0}\right)^{2}}+e^{-\beta\left(\alpha+\alpha_{0}\right)^{2}}\right]
$$

inserting this expression in (8), integrating and fitting the parameters $A, \beta$ and $\alpha_{0}$, we can compare the results with the experimental data from ISR [19]. The results are shown in Fig. 4.

We obtained the parameters for $491 \mathrm{GeV}(A=1.03$, $\alpha_{0}=1.0$ and $\left.\beta=1.0\right)$ and $1030 \mathrm{GeV}\left(A=0.85, \alpha_{0}=1.0\right.$ and $\beta=0.7$ ) incident protons. Our real interest is for a 800 $\mathrm{GeV}$ beam, so, we must interpolate the results. The obtained values were $A=0.93, \alpha_{0}=1.0$ and $\beta=0.85$, and these values will be used in the average polarization calculation.

The transverse rapidity distribution $d \sigma / d \alpha_{t}$ will be as- sumed to be [17],

$$
\frac{1}{\operatorname{ch} \alpha_{t} \operatorname{sh} \alpha_{t}} \frac{d \sigma}{d \alpha_{t}}=A e^{-\beta_{t} \alpha_{t}^{2}},
$$

with $\beta_{t}=6.6$.

The numerical results from the calculation of the average polarization, from Eq (5), to $\bar{\Lambda}$ and $\bar{\Xi}^{+}$are shown in Fig. 5.

We can observe that for $\bar{\Lambda}$ the fit was very good (fact that is independent of $\beta$ ). For $\bar{\Xi}^{+}$we verified the effect of the parameter $\beta$ and included the next resonance, $\bar{\Xi}^{*}(1620)$, that provided a good accord with the experimental data. Calculations of the $\bar{\Sigma}^{-}$polarization are in progress, and preliminary results show that the next resonance, $\bar{\Lambda}^{*}(1520)$ is not so important.

\section{References}

[1] G. Bunce et al., Phys. Rev. Lett. 36, 1113 (1976).

[2] G. Giacomelli, Phys. Rep. 23, 123 (1976).

[3] B. Lundberg et al., Phys. Rev. D40, 3557 (1978).

[4] R. Ramerika et al., Phys. Rev. D33, 3172 (1986).

[5] P.M. Ho et al., Phys. Rev. D44, 3402 (1991).

[6] A. Morelos et al., Phys. Rev. Lett. 71, 2172 (1993).

[7] T. A. DeGrand and H. I. Miettinen, et al., Phys. Rev. D24, 2419 (1981).

[8] J. Soffer and N. A. Törnqvist, Phys. Rev. Lett. 68, 907 (1992).

[9] S. M. Troshin and N. E. Tyurin, Phys. Rev. D55, 1265 (1997).

[10] Y. Hama and T. Kodama, Phys. Rev. D48, 3116 (1993).

[11] C. C. Barros Jr. Doctor thesis, IFUSP (2001).

[12] T. Becher and H. Leutwyler, hep-ph/0103263; Eur. Jour. Phys. C9, 643 (1999).

[13] C. C. Barros Jr. and Y. Hama, Phys. Rev. C63, 065203 (2001).

[14] M. R. Robilotta, Phys. Rev. C63, 0340 (2000).

[15] C. C. Barros Jr. and M. R. Robilotta, hep-ph/0209213.

[16] C. C. Barros Jr., Phys. Rev. D68, 034006 (2003).

[17] Y. Hama and F. S. Navarra, Z. Phys. C53, 501 (1992).

[18] Y. Hama, Nuovo Cim. A46, 569 (1978).

[19] W. Bell et al., Z. Phys. C27, 191 (1985). 